\title{
New Subclass of Meromorphic Functions Associated with Hypergeometric Function
}

Mohamed. A. Khadr

mohamed2019ahmad2020@gmail.com

Department of Mathematics,

College of Computer Science and Mathematics,

University of Mosul, Mosul, Iraq

Received on: 08/12/2020
Ahmed. M. Ali

ahmedgraph@uomosul.edu.iq
Firas Gh. Ahmed

fgahmed@sharjah.ac
Department of Mathematics, College of Sciences,

University of Sharjah, Sharjah,

$U A E$

Accepted on: 05/01/2021

\section{ABSTRACT}

In this paper, we determine sufficient conditions, distortion properties and radii of starlikeness and convexity for functions The hypergeometric meromorphic functions have certain formula in the punctured unit disk which contains in new subclass

Keywords: hypergeometric meromorphic functions, Starlikeness functions, Convexity functions.

\section{Introduction:}

Let $\Sigma$ denote the class of $f(\zeta)$, where $f(\zeta)$ is the meromorphic function normalized

by $f(0)=f^{\prime}(0)-1=0$ and defined by:

$$
f(\zeta)=\frac{1}{\zeta}+\sum_{\kappa=1}^{\infty} a_{\kappa} \zeta^{\kappa}
$$

where (1.1) is analytic in $\mathrm{U}^{*}=\{\zeta: \zeta \in \square$ and $0<|\zeta|<1\}=\mathrm{U} \backslash\{0\}$, and $\square$ be the set of complex numbers, ( $\mathrm{U}^{*}$ is called punctured unit disk),[1].

Two of the most important subclasses of meromorphic functions, namely the starlike and convex functions, but both have very useful analytic characterizations.

Definition 1.1: A function $f$ is said to be convex in $\mathrm{U}^{*}$ if the image of $\mathrm{U}^{*}$ under $f$ is a convex region, i.e."any line segment joining any two points of $f\left(\mathrm{U}^{*}\right)$ lies entirely in $f\left(\mathrm{U}^{*}\right) "$,

[2].

Definition 1.2: Let $\mathrm{U}^{*}$ be the punctured unit disk, if the image of $\mathrm{U}^{*}$ under $f$ is starlike region containing $w=0$, then the function $f$ is called starlike, i.e. "any line segment joining any points of $f\left(\mathrm{U}^{*}\right)$ from $w=0$ lies in $\mathrm{U}^{*} ",[2]$.

We denote the subclasses starlike and convex of order $\chi,(\chi \geq 0)$ in $U^{*}$ by $\Sigma S^{*}(\chi)$ and $\Sigma \mathrm{K}(\chi)$ of $\Sigma$ consisting of all meromorphic functions respectively, the starlike of order $\chi$ satisfy the following condition:

$$
\mathfrak{R}\left(-\frac{\zeta f^{\prime}(\zeta)}{f(\zeta)}\right)>\chi,
$$


the necessary and sufficient condition for a function $f$ to be convex of order $\chi$ can be written as follows [3]:

$$
\mathfrak{R}\left(-1-\frac{\zeta f^{\prime \prime}(\zeta)}{f^{\prime}(\zeta)}\right)>\chi
$$

The functions $f_{m}(\zeta)$ for all $m=1,2$ are defined

$$
f_{m}(\zeta)=\frac{1}{\zeta}+\sum_{\kappa=1}^{\infty} a_{\kappa, m} \zeta^{\kappa}, \quad(m=1,2) .
$$

The convolution of $f_{1}(\zeta)$ and $f_{2}(\zeta)$ defined by:

$$
\left(f_{1} * f_{2}\right)(\zeta)=\frac{1}{\zeta}+\sum_{\kappa=1}^{\infty} a_{\kappa, 1} a_{\kappa, 2} \zeta^{\kappa}
$$

where the convolution is called the Hadamard product.

Consider the function :

$$
\tilde{\emptyset}(\varpi, \sigma ; \zeta)=\frac{1}{\zeta}+\sum_{\kappa=0}^{\infty} \frac{(\varpi)_{\kappa+1}}{(\sigma)_{\kappa+1}} \zeta^{\kappa}, \quad\left(\sigma \in \square / \square_{0}^{-} ; \varpi \in \square\right),
$$

where $\quad(\psi)_{\kappa}:=\frac{\Gamma(\psi+\kappa)}{\Gamma(\psi)}=\left\{\begin{array}{cc}\psi(\psi+1) \ldots(\psi+\kappa-1) & (\kappa=n \in \square ; \psi \in \square), \\ 1 & (\kappa=0 ; \psi \in \square \backslash\{0\}),\end{array}\right.$ and $\square_{0}^{-}=\square^{-} \bigcup\{0\},(\psi)_{\kappa}$ is called Pochhammer symbol and $\Gamma$ is Gamma function, $[4,7$ and 9$]$.

We note that where ${ }_{2} F_{1}(b, \varpi, \sigma ; \zeta)=\sum_{\kappa=0}^{\infty} \frac{(b)_{\kappa}(\varpi)_{\kappa}}{(\sigma)_{\kappa}} \cdot \frac{\zeta^{\kappa}}{\kappa !}$

$\tilde{\emptyset}(\varpi, \sigma ; \zeta)=\frac{1}{\zeta}{ }_{2} F_{1}(1, \varpi, \sigma ; \zeta)$,

is the well-known Gaussin hypergeometric function, [5].

For $f \in \Sigma$ given by (1.1), we reintroduce $c^{t} f(\zeta)$ which is studied by many authors (see for example [6].

$$
c^{t} f(\zeta)=(1-t) f(\zeta)+t \zeta(-f(\zeta))^{\prime}=\frac{1}{\zeta}+\sum_{\kappa=1}^{\infty}(1-(\kappa+1) t) a_{\kappa} \zeta^{\kappa}, \quad(t \geq 0) .
$$

Now, using the convolution between (1.2) and (1.3), we will introduce a new function $M_{\varpi, \sigma}^{t}$ defined on $\Sigma$ by

$$
M_{\varpi, \sigma}^{t} f(\zeta)=\tilde{\emptyset}(\varpi, \sigma ; \zeta) * c^{t} f(\zeta)=\frac{1}{\zeta}+\sum_{\kappa=1}^{\infty} \frac{(\varpi)_{\kappa+1}}{(\sigma)_{\kappa+1}}(1-(\kappa+1) t) a_{\kappa} \zeta^{\kappa}, \quad\left(\zeta \in \mathrm{U}^{*}\right) .
$$

There are many studies about the generalized of meromorphic functions and hypergeometric functions, see [7-11].

For all $\zeta \in \mathrm{U}^{*}$ and $-1 \leq D<S \leq 1$, the function $f \in \Sigma$ is said to be a member of the subclass $\sum_{\sigma, \sigma}^{S, D}(t, \kappa)$ if it satisfies, see[12].

$$
\left|\frac{\zeta\left(M_{\sigma, \sigma}^{t} f(\zeta)\right)^{\prime}+M_{\sigma, \sigma}^{t} f(\zeta)}{D \zeta\left(M_{\sigma, \sigma}^{t} f(\zeta)\right)^{\prime}+S\left(M_{\sigma, \sigma}^{t} f(\zeta)\right)}\right|<1 .
$$

Remark .1.1. It follows from (1.4) that

$$
\zeta\left(M_{\varpi, \sigma}^{t} f(\zeta)\right)^{\prime}=\varpi M_{\varpi+1, \sigma}^{t} f(\zeta)-(\varpi+1) M_{\varpi, \sigma}^{t} f(\zeta) .
$$




\section{Distortion Theorems and Coefficient Estimates}

Theorem 2.1:Let $f$ be the function defined by (1.4) and satisfies (1.5), then, we have

$$
\sum_{\kappa=1}^{\infty} \frac{(\varpi)_{\kappa+1}}{(\sigma)_{\kappa+1}}|(1-(\kappa+1) t)|[(\kappa+1)-(\kappa D+S)]\left|a_{\kappa}\right| \leq S-D,
$$

where $-1 \leq D<S \leq 1$.

Proof: Suppose that (1.5) holds true, then

$$
\begin{aligned}
& \left|\frac{\zeta\left(\frac{-1}{\zeta^{2}}+\sum_{\kappa=1}^{\infty} \frac{(\varpi)_{\kappa+1}}{(\sigma)_{\kappa+1}}(1-(\kappa+1) t) \kappa a_{\kappa} \zeta^{\kappa-1}\right)+\frac{1}{\zeta}+\sum_{\kappa=1}^{\infty} \frac{(\varpi)_{\kappa+1}}{(\sigma)_{\kappa+1}}(1-(\kappa+1) t) a_{\kappa} \zeta^{\kappa}}{D \zeta\left(\frac{-1}{\zeta^{2}}+\sum_{\kappa=1}^{\infty} \frac{(\varpi)_{\kappa+1}}{(\sigma)_{\kappa+1}}(1-(\kappa+1) t) \kappa a_{\kappa} \zeta^{\kappa-1}\right)+S\left(\frac{1}{\zeta}+\sum_{\kappa=1}^{\infty} \frac{(\varpi)_{\kappa+1}}{(\sigma)_{\kappa+1}}(1-(\kappa+1) t) a_{\kappa} \zeta^{\kappa}\right)}\right| \leq 1 \\
& \Rightarrow\left|\frac{\sum_{\kappa=1}^{\infty} \frac{(\varpi)_{\kappa+1}}{(\sigma)_{\kappa+1}}(1-(\kappa+1) t)(\kappa+1) a_{\kappa} \zeta^{\kappa}}{S-D}+\sum_{\kappa=1}^{\infty} \frac{(\varpi)_{\kappa+1}}{(\sigma)_{\kappa+1}}(1-(\kappa+1) t)(\kappa D+S) a_{\kappa} \zeta^{\kappa}\right| \leq 1 \\
& \Rightarrow\left|\sum_{\kappa=1}^{\infty} \frac{(\varpi)_{\kappa+1}}{(\sigma)_{\kappa+1}}(1-(\kappa+1) t)(\kappa+1) a_{\kappa} \zeta^{\kappa}\right|-\left|\frac{S-D}{\zeta}+\sum_{\kappa=1}^{\infty} \frac{(\varpi)_{\kappa+1}}{(\sigma)_{\kappa+1}}(1-(\kappa+1) t)(\kappa D+S) a_{\kappa} \zeta^{\kappa}\right| \leq 0 \\
& \Rightarrow\left|\sum_{\kappa=1}^{\infty} \frac{(\varpi)_{\kappa+1}}{(\sigma)_{\kappa+1}}(1-(\kappa+1) t)(\kappa+1) a_{\kappa} \zeta^{\kappa+1}\right|-\left|S-D+\sum_{\kappa=1}^{\infty} \frac{(\varpi)_{\kappa+1}}{(\sigma)_{\kappa+1}}(1-(\kappa+1) t)(\kappa D+S) a_{\kappa} \zeta^{\kappa+1}\right| \leq 0 \\
& \Rightarrow \sum_{\kappa=1}^{\infty} \frac{(\varpi)_{\kappa+1}}{(\sigma)_{\kappa+1}}|(1-(\kappa+1) t)|(\kappa+1)\left|a_{\kappa}\right|\left|\zeta^{\kappa+1}\right|-S+D-\sum_{\kappa=1}^{\infty} \frac{(\varpi)_{\kappa+1}}{(\sigma)_{\kappa+1}}|(1-(\kappa+1) t)|(\kappa D+S)\left|a_{\kappa}\right|\left|\zeta^{\kappa+1}\right| \leq 0 \\
& \text { when }|\zeta|=r \rightarrow 1 \\
& \sum_{\kappa=1}^{\infty} \frac{(\varpi)_{\kappa+1}}{(\sigma)_{\kappa+1}}|(1-(\kappa+1) t)|[(\kappa+1)-(\kappa D+S)]\left|a_{\kappa}\right| \leq S-D
\end{aligned}
$$

Corollary2.1: Let the function $f$ be defined by (1.4). If $f \in \Sigma_{\varpi, \sigma}^{S, D}(t, \kappa)$, then

$$
\left|a_{\kappa}\right| \leq \frac{(\sigma)_{\kappa+1}(S-D)}{(\varpi)_{\kappa+1}|(1-(\kappa+1) t)|[(\kappa+1)-(\kappa D+S)]}, \quad(\kappa \geq 1) .
$$

\#

The sharp for functions $f(\zeta)$ at the term $\kappa$ has the form:

$$
f_{\kappa}(\zeta)=\frac{1}{\zeta}+\frac{(\sigma)_{k+1}(S-D)}{(\varpi)_{\kappa+1} \mid(1-(\kappa+1) t \mid[(\kappa+1)-(\kappa D+S)]} \zeta^{\kappa}, \quad(\kappa \geq 1) .
$$

Corollary2.2: If $t=0, S=1$ and $D=-1$ in Theorem 2.1, then $f \in \Sigma$ satisfying the following 
condition

$$
\sum_{\kappa=1}^{\infty} \frac{(\varpi)_{\kappa+1}}{(\sigma)_{\kappa+1}} \kappa\left|a_{\kappa}\right| \leq 1
$$

and it is starlike in $\mathrm{U}^{*}$.

$$
\#
$$

Corollary 2.3: For $S=1$ and $D=-1$ in Theorem 2.1, we have

$$
\sum_{\kappa=1}^{\infty} \frac{(\varpi)_{\kappa+1}}{(\sigma)_{\kappa+1}}|(1-(\kappa+1) t)| \kappa\left|a_{\kappa}\right| \leq 1
$$

and therefore the function $M_{\sigma, \sigma}^{t} f(\zeta)$ is starlike in $\mathrm{U}^{*}$.

Corollary 2.4: If $t=0, S=1$ and $D=-1$ in Theorem 2.1, then $f \in \Sigma$ satisfying the following condition

$$
\sum_{\kappa=1}^{\infty} \frac{(\varpi)_{\kappa+1}}{(\sigma)_{\kappa+1}} \kappa^{2}\left|a_{\kappa}\right| \leq 1
$$

and it is convex in $\mathrm{U}^{*}$.

$$
\text { \# }
$$

Corollary 2.5: For $S=1$ and $D=-1$ in Theorem 2.1, we have

$$
\sum_{\kappa=1}^{\infty} \frac{(\varpi)_{\kappa+1}}{(\sigma)_{\kappa+1}}|(1-(\kappa+1) t)| \kappa^{2}\left|a_{\kappa}\right| \leq 1
$$

and therefore the function $M_{\sigma, \sigma}^{t} f(\zeta)$ is convex in $\mathrm{U}^{*}$.

The following Theorem is given the distortion property of function in the subclass $\Sigma_{\varpi, \sigma}^{S, D}(t, \kappa)$.

Theorem2.2:The function $f$ defined by (1.4) in the subclass $\Sigma_{\varpi, \sigma}^{S, D}(t, \kappa)$, then for all $0<|\zeta|=r<1$, we have

$$
\frac{1}{r}-\frac{(S-D)}{(2-(S+D))} r \leq|f(\zeta)| \leq \frac{1}{r}+\frac{(S-D)}{(2-(S+D))} r
$$

and

$$
\frac{1}{r^{2}}-\frac{(S-D)}{(2-(S+D))} \leq\left|f^{\prime}(\zeta)\right| \leq \frac{1}{r^{2}}+\frac{(S-D)}{(2-(S+D))}
$$

with equality for

$$
f(\zeta)=\frac{1}{\zeta}+\frac{(S-D)}{(2-(S+D))} \zeta .
$$

Proof: Let $f \in \sum_{\sigma, \sigma}^{S, D}(t, \kappa)$. Then, Theorem 2.1 readily yields the inequality

$$
\sum_{\kappa=1}^{\infty} \frac{(\varpi)_{\kappa+1}}{(\sigma)_{\kappa+1}}|(1-(\kappa+1) t)|\left|a_{\kappa}\right| \leq \frac{S-D}{[(\kappa+1)-(\kappa D+S)]}
$$

For $0<|\zeta|=r<1$, and making use of (2.6), we have

$$
\begin{aligned}
|f(\zeta)| & \leq \frac{1}{|\zeta|}+\sum_{\kappa=1}^{\infty} \frac{(\varpi)_{\kappa+1}}{(\sigma)_{\kappa+1}}|(1-(\kappa+1) t)|\left|a_{\kappa}\right||\zeta|^{\kappa} \leq \frac{1}{r}+r \sum_{\kappa=1}^{\infty} \frac{(\varpi)_{\kappa+1}}{(\sigma)_{\kappa+1}} \mid\left(1-(\kappa+1) t|| a_{\kappa} \mid\right. \\
& \leq \frac{1}{r}+r \frac{S-D}{[(\kappa+1)-(\kappa D+S)]} \leq \frac{1}{r}+\frac{(S-D)}{(2-(S+D))} r
\end{aligned}
$$


and the other side of the inequality in (2.4) can be obtained using similar procedure.

For (2.5), again from Theorem 2.1, it follows that

$$
\sum_{\kappa=1}^{\infty} \frac{(\varpi)_{\kappa+1}}{(\sigma)_{\kappa+1}}|(1-(\kappa+1) t)| \kappa\left|a_{\kappa}\right| \leq \frac{\kappa(S-D)}{[(\kappa+1)-(\kappa D+S)]} .
$$

Hence

$$
\begin{aligned}
\left|f^{\prime}(\zeta)\right| \leq \frac{1}{\left|\zeta^{2}\right|}+\sum_{\kappa=1}^{\infty} \frac{(\varpi)_{\kappa+1}}{(\sigma)_{\kappa+1}}|(1-(\kappa+1) t)| \kappa\left|a_{\kappa}\right||\zeta|^{\kappa-1} & \leq \frac{1}{r^{2}}+\frac{\kappa(S-D)}{[(\kappa+1)-(\kappa D+S)]} \\
& \leq \frac{1}{r^{2}}+\frac{(S-D)}{(2-(S+D))} .
\end{aligned}
$$

By similarity, the other side of the inequality follows and the proof is complete. \# 


\section{Convexity and Radii of Starlikeness}

Theorem3.1:If the function $f$ in the subclass $\Sigma_{\sigma, \sigma}^{S, D}(t, \kappa)$, then $f$ is meromorphically starlike of order $\chi(0 \leq \chi<1)$ in $|\zeta|<r_{1}$,

$$
r_{1}=r_{1}(S, D, \kappa)=\inf _{\kappa \geq 1}\left\{\frac{(1-\chi)[(\kappa+1)-(\kappa D+S)]}{(\kappa+2-\chi)(S-D)}\right\}^{\frac{1}{\kappa+1}}
$$

The sharp of the function $f_{\kappa}$ given by (2.3).

Proof:It suffices to prove that

$$
\left|\frac{\zeta\left(M_{\sigma, \sigma}^{t} f(\zeta)\right)^{\prime}}{M_{\sigma, \sigma}^{t} f(\zeta)}+1\right| \leq 1-\chi
$$

for $|\zeta|<r_{1}$, we have

$$
\begin{aligned}
\left|\frac{\zeta\left(M_{\varpi, \sigma}^{t} f(\zeta)\right)^{\prime}}{M_{\varpi, \sigma}^{t} f(\zeta)}+1\right| & =\mid \frac{\frac{-1}{\zeta}+\sum_{\kappa=1}^{\infty} \frac{(\varpi)_{\kappa+1}}{(\sigma)_{\kappa+1}}(1-(\kappa+1) t) \kappa a_{\kappa} \zeta^{\kappa}}{\frac{1}{\zeta}+\sum_{\kappa=1}^{\infty} \frac{(\varpi)_{\kappa+1}}{(\sigma)_{\kappa+1}}(1-(\kappa+1) t) a_{\kappa} \zeta^{\kappa}}+1 \\
& =\left|\frac{\sum_{\kappa=1}^{\infty} \frac{(\varpi)_{\kappa+1}}{(\sigma)_{\kappa+1}}(1-(\kappa+1) t) a_{\kappa} \zeta^{\kappa}(\kappa+1)}{\frac{1}{\zeta}+\sum_{\kappa=1}^{\infty} \frac{(\varpi)_{\kappa+1}}{(\sigma)_{\kappa+1}}(1-(\kappa+1) t) a_{\kappa} \zeta^{\kappa}}\right| \\
& \leq \frac{\sum_{\kappa=1}^{\infty} \frac{(\varpi)_{\kappa+1}}{(\sigma)_{\kappa+1}}|(1-(\kappa+1) t)|\left|a_{\kappa}\right|(\kappa+1)|\zeta|^{\kappa}}{|\zeta| \sum_{\kappa=1}^{\infty} \frac{(\varpi)_{\kappa+1}}{(\sigma)_{\kappa+1}}|(1-(\kappa+1) t)|\left|a_{\kappa}\right||\zeta|^{\kappa}} \\
& \leq \frac{\sum_{\kappa=1}^{\infty} \frac{(\varpi)_{\kappa+1}}{(\sigma)_{\kappa+1}}|(1-(\kappa+1) t)|\left|a_{\kappa}\right|(\kappa+1)|\zeta|^{\kappa+1}}{1-\sum_{\kappa=1}^{\infty} \frac{(\varpi)_{\kappa+1}}{(\sigma)_{\kappa+1}}|(1-(\kappa+1) t)|\left|a_{\kappa}\right||\zeta|^{\kappa+1}} .
\end{aligned}
$$

Hence, (3.3) holds true if

$\sum_{\kappa=1}^{\infty} \frac{(\varpi)_{\kappa+1}}{(\sigma)_{\kappa+1}}|(1-(\kappa+1) t)|\left|a_{\kappa}\right|(\kappa+1)|\zeta|^{\kappa+1} \leq(1-\chi)\left(1-\sum_{\kappa=1}^{\infty} \frac{(\varpi)_{\kappa+1}}{(\sigma)_{\kappa+1}}|(1-(\kappa+1) t)|\left|a_{\kappa}\right||\zeta|^{\kappa+1}\right)$ (3.4)

or

$$
\begin{aligned}
& \sum_{\kappa=1}^{\infty} \frac{(\varpi)_{\kappa+1}}{(\sigma)_{\kappa+1}}|(1-(\kappa+1) t)|\left|a_{\kappa}\right||\zeta|^{\kappa+1} \frac{(\kappa+2-\chi)}{(1-\chi)} \leq 1 \\
& \frac{(\varpi)_{\kappa+1}}{(\sigma)_{\kappa+1}}|(1-(\kappa+1) t)|\left|a_{\kappa}\right||\zeta|^{\kappa+1} \frac{(\kappa+2-\chi)}{(1-\chi)} \leq 1, \text { for } \kappa \geq 1
\end{aligned}
$$

with the aid of (2.2) and (3.5) is true if, for all $\kappa \geq 1$, 


$$
\frac{(\varpi)_{\kappa+1}}{(\sigma)_{\kappa+1}}|(1-(\kappa+1) t) \| \zeta|^{\kappa+1} \frac{(\kappa+2-\chi)}{(1-\chi)} \leq \frac{\frac{(\varpi)_{\kappa+1}}{(\sigma)_{\kappa+1}}|(1-(\kappa+1) t)|[(\kappa+1)-(\kappa D+S)]}{S-D} .
$$

Solving (3.6) for $|\zeta|$, we obtain

$$
|\zeta| \leq\left\{\frac{(1-\chi)[(\kappa+1)-(\kappa D+S)]}{(\kappa+2-\chi)(S-D)}\right\}^{\frac{1}{\kappa+1}}, \quad \forall \kappa \geq 1 .
$$

\#

Theorem3.2:The function $f$ which defined by (1.4) in the subclass $\Sigma_{\sigma, \sigma}^{S, D}(t, \kappa)$, then $f$ is meromorphically convex of order $\chi(0 \leq \chi<1)$ in $|\zeta|<r_{2}$, where

$$
r_{2}=r_{2}(S, D, \kappa)=\inf _{k \geq 1}\left\{\frac{(1-\chi)[(\kappa+1)-(\kappa D+S)]}{\kappa(\kappa+2-\chi)(S-D)}\right\}^{\frac{1}{\kappa+1}}
$$

The sharp of the function at $\kappa$, i.e. $f_{\kappa}$ is given by (2.3).

Proof:From proof of Theorem 3.1, we can show that

$$
\left|\frac{\zeta\left(M_{\sigma, \sigma}^{t} f(\zeta)\right)^{\prime \prime}}{\left(M_{\sigma, \sigma}^{t} f(\zeta)\right)^{\prime}}+2\right| \leq(1-\chi),
$$

for $|\zeta|<r_{2}$, with the aid of Theorem 2.1. Thus, we have the assertion of Theorem 3.2.

\section{Convex Linear Combinations}

The next results involves a linear combination of several functions of the type (2.3).

Theorem 4.1. Let

$$
f_{0}(\zeta)=\frac{1}{\zeta}
$$

and

$$
f_{\kappa}(\zeta)=\frac{1}{\zeta}+\frac{(\sigma)_{\kappa+1}(S-D)}{(\varpi)_{\kappa+1} \mid(1-(\kappa+1) t \mid[(\kappa+1)-(\kappa D+S)]} \zeta^{\kappa} \quad(\kappa \geq 1) .
$$

Then $f \in \Sigma_{\varpi, \sigma}^{S, D}(t, \kappa)$ if and only if it can be expressed in the form

$$
f(\zeta)=\sum_{\kappa=0}^{\infty} \eta_{\kappa} f_{\kappa}(\zeta)
$$

where $\eta_{\kappa} \geq 0$ and $\sum_{\kappa=0}^{\infty} \eta_{\kappa}=1$.

Proof. From (4.1), (4.2) and (4.3), it is easily seen that

$$
f(\zeta)=\sum_{\kappa=0}^{\infty} \eta_{\kappa} f_{\kappa}(\zeta)=\frac{1}{\zeta}+\sum_{\kappa=1}^{\infty} \frac{(\sigma)_{\kappa+1}(S-D) \eta_{\kappa}}{(\varpi)_{\kappa+1} \mid(1-(\kappa+1) t \mid[(\kappa+1)-(\kappa D+S)]} \zeta^{\kappa}
$$

since

$$
\sum_{\kappa=1}^{\infty} \frac{(\varpi)_{\kappa+1} \mid(1-(\kappa+1) t \mid[(\kappa+1)-(\kappa D-S)]}{(\sigma)_{\kappa+1}(S-D)} \cdot \frac{\eta_{\kappa}(\sigma)_{\kappa+1}(S-D)}{(\varpi)_{\kappa+1} \mid(1-(\kappa+1) t \mid[(\kappa+1)-(\kappa D-S)]}
$$




$$
=\sum_{\kappa=1}^{\infty} \eta_{\kappa}=1-\eta_{0} \leq 1,
$$

it follows from Theorem 2.1 that the function $f \in \Sigma_{\sigma, \sigma}^{S, D}(t, \kappa)$.

Conversely, let us suppose that $f \in \Sigma_{\varpi, \sigma}^{S, D}(t, \kappa)$. Since

Setting

$$
\left|a_{\kappa}\right| \leq \frac{(\sigma)_{\kappa+1}(S-D)}{(\varpi)_{\kappa+1}|(1-(\kappa+1) t)|[(\kappa+1)-(\kappa D+S)]}, \quad(\kappa \geq 1) .
$$

and

$$
\eta_{\kappa}=\frac{(\varpi)_{\kappa+1}|(1-(\kappa+1) t)|[(\kappa+1)-(\kappa D+S)]}{(\sigma)_{\kappa+1}(S-D)}, \quad(\kappa \geq 1)
$$

$$
\eta_{0}=1-\sum_{\kappa=1}^{\infty} \eta_{\kappa}
$$

it follows that $f(\zeta)=\sum_{\kappa=0}^{\infty} \eta_{\kappa} f_{\kappa}(\zeta)$.

\#

Theorem 4.2. The subclass $\Sigma_{\sigma, \sigma}^{S, D}(t, \kappa)$ is closed under convex linear combinations.

Proof.Suppose that the functions $f_{1}(\zeta)$ and $f_{2}(\zeta)$ defined by

$$
f_{v}(\zeta)=\frac{1}{\zeta}+\sum_{\kappa=1}^{\infty} a_{\kappa, v} \zeta^{\kappa}, \quad\left(v=1,2 ; \zeta \in \mathrm{U}^{*}\right)
$$

are in the subclass $\sum_{\sigma, \sigma}^{S, D}(t, \kappa)$.

Setting

$$
f(\zeta)=\mu f_{1}(\zeta)+(1-\mu) f_{2}(\zeta), \quad(0 \leq \mu \leq 1) .
$$

We find from (4.5) that

$$
f(\zeta)=\frac{1}{\zeta}+\sum_{\kappa=1}^{\infty} \frac{(\varpi)_{\kappa+1}}{(\sigma)_{\kappa+1}}(1-(\kappa+1) t)\left\{\mu a_{\kappa, 1}+(1-\mu) a_{\kappa, 2}\right\} \zeta^{\kappa} \quad\left(0 \leq \mu \leq 1 ; \zeta \in \mathrm{U}^{*}\right) .
$$

In view of Theorem 2.1, we have

$$
\begin{aligned}
& \sum_{\kappa=1}^{\infty} \frac{(\varpi)_{\kappa+1}}{(\sigma)_{\kappa+1}}(1-(\kappa+1) t)[(\kappa+1)-(\kappa D+S)]\left(\mu a_{\kappa, 1}+(1-\mu) a_{\kappa, 2}\right) \\
&=\mu \sum_{\kappa=1}^{\infty} \frac{(\varpi)_{\kappa+1}}{(\sigma)_{\kappa+1}}(1-(\kappa+1) t)[(\kappa+1)-(\kappa D+S)] a_{\kappa, 1} \\
&+(1-\mu) \sum_{\kappa=1}^{\infty} \frac{(\varpi)_{\kappa+1}}{(\sigma)_{\kappa+1}}(1-(\kappa+1) t)[(\kappa+1)-(\kappa D+S)] a_{\kappa, 2} \\
& \leq \mu(S-D)+(1-\mu)(S-D)=(S-D) .
\end{aligned}
$$

Which shows that $f \in \sum_{\sigma, \sigma}^{S, D}(t, \kappa)$. 


\section{REFERENCES}

[1] Zhi-Gang Wang, Zhi-Hong Liu and Yong Sun, Some subclasses of meromorphic functions Associated with a Family of Integral operators, Journal of Inequalities and Applications, 27(2009), 18 pages.

[2] A. W. Goodman, Univalent Functions. USA: University Of South Florida, 1983.

[3] M. Nunokawa and O. P. Ahuja, On meromorphic starlike and convex functions, Indian J. Pure Appl. Math., 32(7)(2001), pp. 1027-1032.

[4] N. E. Cho and I. H. Kim, Inclusion properties of certain classes of meromorphic functions associated with the generalized hypergeometric function, Appl. Math. And Computer, 187, (2007), 115-121.

[5] F. Ghanim and M. Darus, Subordinations for Analytic Functions Defined by the Linear Operator, American Journal of Scientific Research, 2 (2009), 52-59.

[6] A. K. Mishra, T. Panigrahi and R. K. Mishra, subordination and inclusion theorems for subclasses of meromorphic functions with applications to electromagnetic cloaking, Mathematical and Computer Modelling, 57 (2013), 945-962.

[7] J. Dziok, H.M. Srivastava, Some subclasses of analytic functions with fixed argument of coefficients associated with the generalized hypergeometric function, Adv. Stud. Contemp. Math ., 5 (2002), 115-125.

[8] J. Dziok, H.M. Srivastava, Certain subclasses of analytic functions associated with the neralized hypergeometric function, Trans. Spec. Funct., 14 (2003), 718.

[9] F. Ghanim and M.Darus, New subclass of multivalent hypergeometric meromorphic functions, International Journal of Pure and Applied Mathematics, 61 (3), 2010, 269-

280.

[10] F. Ghanim and M.Darus, On certain subclass of meromorphic univalent functions with fixed residue $\alpha$, Far East J. Math. Sci., 26, No. 1 (2007), 195207.

[11] F Ghanim, M Darus and S Sivasubramanian, On new subclass of analytic univalent function, International Journal of Pure and Applied Mathematics, 40 (3), 2007, 307-319.

[12] M. Albehbah and M. Darus, New subclass of multivalent hypergeometric meromorphic functions, Kragujevac Journal of Mathematics, 42(1), 2018, 83-95. 